\title{
Analysis of metallothionein and vimentin immunoreactivity in pharyngeal squamous cell carcinoma and its microenvironment
}

\author{
Magdalena Dutsch-Wicherek • Agata Lazar • \\ Romana Tomaszewska • Wojciech Kazmierczak • \\ Lukasz Wicherek
}

Received: 18 August 2012 / Accepted: 10 January 2013 /Published online: 9 February 2013

(C) The Author(s) 2013. This article is published with open access at Springerlink.com

\begin{abstract}
Metallothionein (MT) has been shown to have pro-proliferative anti-apoptotic activity and to be involved in microenvironment remodeling. The aim of this study has been to determine whether the changes in MT and vimentin immunoreactivity observed in cancer and its microenvironment are related to the local spread of the disease. The immunoreactivity levels of both MT and vimentin were evaluated together with CD56 and CD57 antigens in 49 tissue samples taken from patients with squamous cell carcinoma originating from the palatine tonsils and in 20 tissue
\end{abstract}

\footnotetext{
M. Dutsch-Wicherek $(\bowtie)$

Outpatient Unit of Otolaryngology,

Lukaszczyk Oncological Center,

Bydgoszcz, Poland

e-mail: mowicher@gmail.com
}

A. Lazar $\cdot$ R. Tomaszewska

The Department of Pathomorphology, Jagiellonian University,

Krakow, Poland

W. Kazmierczak

Department of Otolaryngology and Oncological Laryngology

with Subdivision of Audiology and Phoniatry,

Jurasz's University Hospital,

Bydgoszcz, Poland

W. Kazmierczak

Department of Pathophisiology of Hearing and Balance System, Ludwik Rydygier's Collegium Medicum,

Nicolaus Copernicus University,

Bydgoszcz, Poland

\section{Wicherek}

Department of Gynecology and Oncology and Gynecological

Nursing, Ludwik Rydygier Collegium Medicum,

Nicolaus Copernicus University,

Bydgoszcz, Poland samples derived from patients with chronic tonsillitis (the reference group). MT immunoreactivity levels were statistically significantly higher in the tissue samples from squamous cell carcinoma than in those of the reference group and also higher in the squamous cell carcinoma samples compared with the stromal samples. Moreover, stromal fibroblasts exhibited high vimentin and MT immunoreactivity levels. Statistically significantly higher MT immunoreactivity levels within the tumor cells were identified in patients with the presence of lymph node metastases in contrast to those patients without such metastases. Vimentin was detected in both the tumor and the stromal tissue samples and presented an interesting pattern of staining strongly expressed within the stroma and the septal architecture of the tumor. The number of CD56- and CD57-positive lymphocytes identified in tissue samples both from squamous cell carcinoma and from the stroma was statistically significantly lower than that in the reference group. MT expression by tumor cells is thus associated with an aggressive phenotype of the tumor and the ability to create metastases.

Keywords Metallothionein (MT) · Vimentin · Cancer microenvironment $\cdot$ Cancer-associated fibroblasts $\cdot$ Human

\section{Introduction}

Tumor microenvironment remodeling is a response to tumor growth and aggressiveness and seems to play a crucial role in both local tumor spread and the process of creating metastases (Witz 2009; Dvorak 1986; Lorusso and Ruegg 2008). Moreover, tumor microenvironment remodeling is a complex process that includes angiogenesis, the epithelial to mesenchymal transition and a change in the activity profile 
of various cells, such as those of the immune system and structural cells, which, like fibroblasts, can be activated into cancer-associated fibroblasts (CAFs). The cells of the tumor microenvironment that are most important for the formation of metastases are endothelial cells, tumor-associated macrophages and CAFs (Witz 2009; Dvorak 1986; Lorusso and Ruegg 2008; Acevedo et al. 2007).

Metastatic tumor cells must first acquire the ability to detach from the tumor and enter the vessels, migrate and home toward the distant tissues forming the secondary tumors. This ability is linked with the epithelial to mesenchymal transition and the process is characterized at the molecular level by the expression of mesenchymal markers including vimentin and N-cadherin. Vimentin is a cytoskeletal intermediate filament that is also expressed by CAF (Hugo et al. 2007; Xu et al. 2006; Grunert et al. 2003). These mesenchymal markers appear shortly before tumor invasion and degrade the basal membrane and extracellular matrix by means of secreted serine proteases and matrix metalloproteinases. CAFs express both IGF (insulin-growth factor) and HGF (hepatocyte growth factor), which stimulate cell survival, migration and the secretion of proangiogenic factors, such as FGF-2 (fibroblast growth factor-2), vascular endothelial growth factor and proinflammatory cytokines, such as interleukin-1 (IL-1), IL-6, IL-8 and tumor necrosis factor- $\alpha$ (Hugo et al. 2007; Xu et al. 2006; Grunert et al. 2003). These cells not only induce their own migration to the tumor but also promote the survival, proliferation and invasion of tumor cells and angiogenesis, in addition to improving the ability of the tumor to disseminate (Orimo et al. 2005; Hayward et al. 2001; De Wever and Mareel 2005). The expression of vimentin in various cancers has been related to poor prognostic factors, as, in prostate cancer, vimentin is overexpressed and contributes to its invasive and metastatic potential and, in non-small-cell lung cancer, vimentin overexpression has been found to be an independent prognostic factor for poor survival (Satelli and Li 2011; de Araujo et al. 1993).

Metallothioneins (MT) are a family of low-molecularweight proteins that perform various important activities. MT expression in cell cytoplasm protects against cytotoxicity, whereas nuclear expression protects against genotoxicity (Cherian et al. 2003; Apostolova and Cherian 2000) and has been shown to protect against oxidative stress (Theocharis et al. 2004). MT also has an important antiapoptotic and pro-proliferative activity (Klaassen et al. 1999; Shimoda et al. 2003). Sundelin et al. (1997) have observed MT expression in cancers in which it has been associated with both higher cell proliferation rates and fewer positive apoptotic cells (Jayasurya et al. 2000; Sundelin et al. 1997). Moreover, MT plays an important immunomodulating role (Sundelin et al. 1997). In our previous studies, MT has been observed in patients with head and neck cancer and breast adenocarcinoma and has been found in the cancer microenvironment. In salivary gland adenocarcinoma, MT expression has been detected in both the cancer and in the stroma in which it is represented by stromal fibroblasts (Dutsch-Wicherek et al. 2005, 2008, 2010; Popiela et al. 2006). The tumor modifies the local anti-tumor immune response, which creates the suppressive tumor microenvironment. The two populations of lymphocytes, namely NKTs (natural killer-like T cells) and CD56- and CD57expressing cells, have an important immunoregulatory role (Okada et al. 1995; Takii et al. 1994). CD56-positive T lymphocytes demonstrate an effector function of cytotoxic T cells (Satoh et al. 1996), whereas CD57-positive T lymphocytes demonstrate an immunoregulatory role for Tcytotoxic lymphocytes (Kuss et al. 2004; Sallusto et al. 2004). Since the immunohistochemical method does not allow the type of immune cells to be identified precisely, our goal has been to determine the presence of CD56- and CD57-positive cells responsible for the effector immune response in pharyngeal carcinoma and its stroma.

We have previously demonstrated that MT and vimentin are proteins involved in the remodeling of the tumor microenvironment in salivary gland adenocarcinomas (DutschWicherek et al. 2010). The aim of our present study has been to determine whether the changes in MT and vimentin expression observed in tumor microenvironment remodeling in adenocarcinoma tissue samples are typical for this histological type of tumor only or whether they are found in the tissue of growing tumors regardless of histological type.

\section{Materials and methods}

\section{Group of patients}

For our study, we recruited patients with primary squamous cell carcinoma originating from the palatine tonsils and selected 49 from each of whom a tissue sample from the carcinoma and from the tumor microenvironment were analyzed. The microenvironment or stroma of the tumor was defined as the surrounding tissue with an area of $1 \mathrm{~cm}^{2}$ macroscopically and histologically free of cancer infiltration, with the distance from the tumor front not exceeding $1 \mathrm{~cm}$.

In all the patients, radical surgery of the tumor was performed together with lymphadenectomy. In each case, the patient's consent was obtained. Additionally, approval for the research program was granted from the Ethical Committee of the Jagiellonian University in Krakow: KBET/90/B/2005.

All the tissue samples were histopathologically verified. Pathological analysis with classical hematoxylin and eosin staining techniques after fixation of the surgically removed material in formalin was performed in the Pathology Department of the Jagiellonian University by two experienced 
pathologists (R.T. and A.L.) who worked independently. In detail, the tissue was fixed in a solution of $10 \%$ formalin, rinsed, dehydrated and transferred through a progressively increasing concentration of ethanol (from 50\% to absolute alcohol) and a series of xylenes (I-III) to molten paraffin wax. Finally, the tissue blocks were sectioned and the sections were placed onto glass slides. The process was mainly automated but both the paraffin embedding and the cutting of the tissue samples into 3to 4- $\mu \mathrm{m}$-thick sections were performed manually.

The characteristics of the patient group are presented in Table 1.

\section{Reference group}

As a reference group, we chose to collect palatine tonsils that had been removed from patients because of recurrent tonsillitis. In these tissue samples, we evaluated the epithelium lining the tonsils rather than the lymphoid tissue (Table 2).

\section{Immunohistochemical analysis}

In the present study, we aimed to analyze the immunoreactivity levels of various antigens in pharyngeal squamous cell

Table 1 Characteristics of the patient group and their tumors

\begin{tabular}{ll}
\hline Squamous cell carcinoma of palatine tonsils & Number \\
\hline Patients & \\
Total number & 49 \\
Men & 35 \\
Women & 14 \\
Age range (average) in years & $31-80(55.8)$ \\
Men & $31-80(55.5)$ \\
Women & $41-79(57.9)$ \\
Stage & \\
I & 1 \\
II & 16 \\
III & 11 \\
IV & 21 \\
Presence of lymph node metastases & \\
Absent & 15 \\
Present & 34 \\
T stage & \\
T1 & 2 \\
T2 & \\
T3 & 32 \\
T4 & 9 \\
Tumor grade & \\
G1 & \\
G2 & \\
G3 & \\
\hline & \\
&
\end{tabular}

Table 2 Characteristics of the reference group

\begin{tabular}{ll}
\hline Palatine tonsils & Number \\
\hline Total number & 20 \\
Men & 9 \\
Women & 11 \\
Age range (average) in years & $16-57(30.41)$ \\
Men & $20-53(33.37)$ \\
Women & $16-57(27.7)$ \\
\hline
\end{tabular}

carcinoma and its stroma. We were also interested in the distribution of the antigen immunoreactivity throughout the tissue of the tumor, including the tumor stroma. For this reason, we chose the immunohistochemical method for the present study. This is also the only method that shows the actual architecture of the interaction between the tumor and its stroma.

From every tumor, 3-4 tissue samples were taken (depending on tumor size; if, for instance, the tumor was $1 \mathrm{~cm}$ in diameter, then it was subject to further analysis). Every tissue sample was embedded in paraffin and formed into a tissue block, which was then cut into sections. All the tissue sections were further histopathologically verified and immunohistochemistry was performed.

Two observers, working independently and having no knowledge of the clinicopathological data, reviewed the immunohistochemical expression of MT, vimentin and CD56- and CD57-positive cells. Immunohistochemical analysis was performed in the Pathology Department of the Jagiellonian University. Sections $(5 \mu \mathrm{m}$ thick) from each case were deparaffinized, rehydrated and rinsed in distilled water. Endogenous peroxidase activity was blocked by an 8 -min incubation in $3 \% \mathrm{H}_{2} \mathrm{O}_{2}$ at room temperature. The sections were then rinsed and immersed in a boiling citrate buffer $(\mathrm{pH} 6.0)$ in a microwave oven with three changes of buffer for 5 min each. Immunohistochemistry was performed in each case by applying the Envision method and a DakoAutostainer. The samples were stained automatically, with immunohistochemical staining being based on the antigen-antibody reaction. Microscopy was performed by using an Axio Zeiss microscope and the tissue sections were then evaluated under both $20 \times$ and $40 \times$ magnification.

The following antibodies were applied: CD56, CD57 (Novocastra; dilution 1:50) Vimentin (DAKO, Denmark; dilution 1:50) and MT (ABCAM; dilution 1:25) for $10 \mathrm{~min}$ at room temperature. Sections were counterstained with hematoxylin and mounted in glycergel. As a positive control, a tonsil specimen was taken for MT immunostaining. All staining was performed identically, with only the primary antibody being omitted as a negative control. Expression for MT and for vimentin was evaluated in entire sections, 
Fig. 1 Immunoreactivity (brown) of CD56 (a) and CD57 (b) antigens in squamous cell carcinoma and its stroma; note the cytoplasmic staining pattern. Magnification $10 \times$ Bars $50 \mu \mathrm{m}$
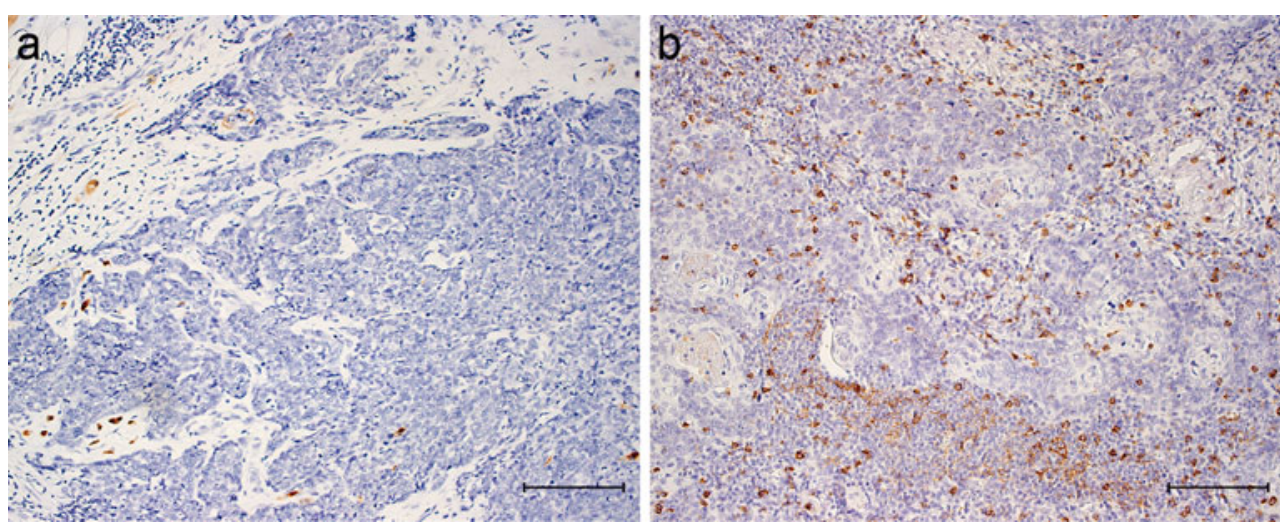

both in the area of the tumor and in the stroma, as follows: 0 , no reactivity; +1 , weak cytoplasmic staining pattern (also granular in the paranuclear region) in up to $10 \%$ of positive cells; +2 , marked cytoplasmic (sometimes together with membranous) staining in $11 \%-30 \%$ of the cells; +3 , high expression in more than $30 \%$ of positive cells. Variable scales were used to evaluate the amount of CD56+ and CD57+ cells semiquantitatively, depending on their general number in the specimen and provided an average cell number per $1 \mathrm{hpf}$ (high power field, objective magnification $\times 40$ ). CD56+ and $\mathrm{CD} 57+$ cells were thus estimated as follows: 0 , lack of positive cells; +1 , single positive cells in the specimen; $+2,2-5$ positive cells per $1 \mathrm{hpf} ;+3$, more than 5 positive cells/ $1 \mathrm{hpf}$.

\section{Statistical analysis}

The distribution of variables in the study groups of the patients, as checked with the use of the Shapiro-Wilk test, showed that each of the patients was indeed different from normal. The statistical significance between the groups was determined by the Kruskal-Wallis test, a one-way analysis of variance by ranks. The Mann-Whitney $U$ test was then used as applicable. All statistical analyses were carried out with the Statistica 8.0 software program. A $P$-value of $<0.05$ was considered as indicative of statistical significance.

\section{Results}

The immunoreactivity levels of the various antigens in the squamous cell carcinoma and stromal tissue samples were as follows.

\section{CD56 and CD57 immunoreactivity}

CD56 antigen immunoreactivity was identified in $4 \%$ of cells in tissue samples from squamous cell carcinoma and in $4 \%$ of cells from the stromal samples and presented a cytoplasmic pattern of staining. CD57 antigen immunoreactivity was observed in $27.5 \%$ of cells of tissue samples from squamous cell carcinoma and in $16 \%$ of cells from the stromal tissue samples and also presented a cytoplasmic pattern of staining (Fig. 1).

\section{MT immunoreactivity}

MT antigen immunoreactivity was observed in $84 \%$ of the tissue samples from squamous cell carcinoma and in $21.7 \%$ of the stromal tissue samples and most frequently presented a nuclear-cytoplasmic pattern of staining. The highest MT immunoreactivity levels were observed in the tissue samples from squamous cell carcinoma derived from patients with the presence of lymph node metastases
Fig. 2 Strong MT immunoreactivity (brown) in squamous cell carcinoma of a patient with lymph node metastases. Magnification 10× (a), $40 \times$ (b). Bars $50 \mu \mathrm{m}$
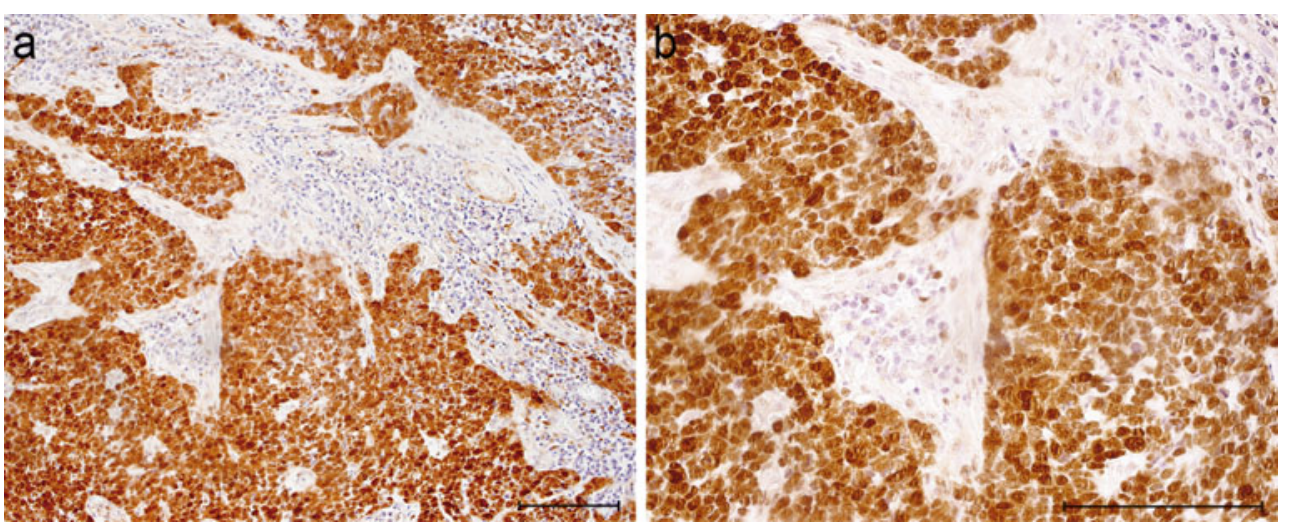
Fig. 3 Immunoreactivity of MT antigen (brown) in stromal fibroblasts. Magnification 10× (a), $40 \times$ (b). Bars $50 \mu \mathrm{m}$
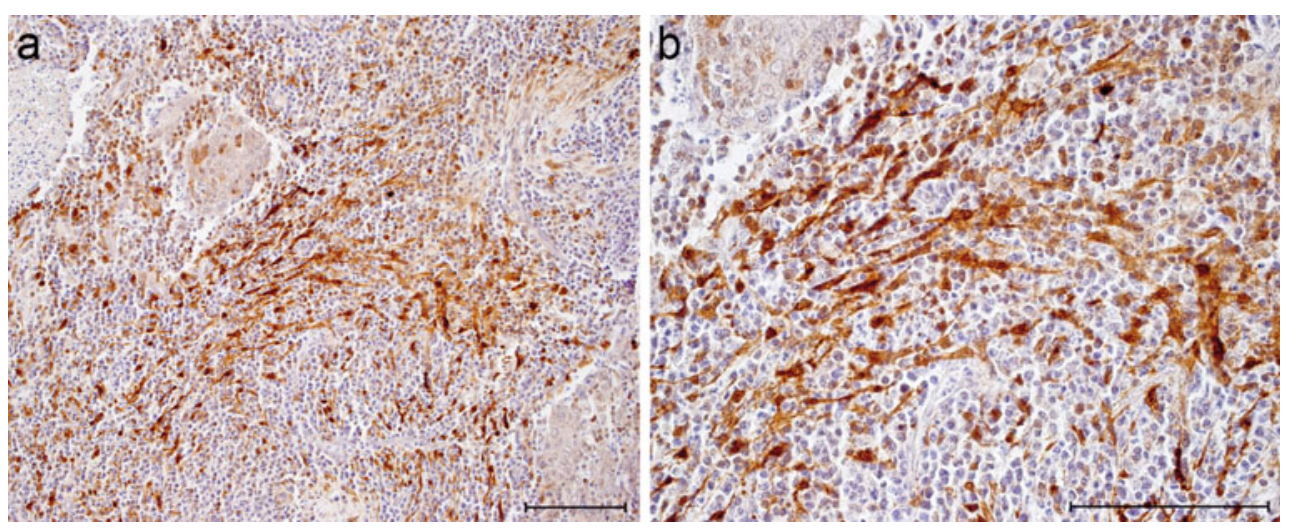

(Fig. 2a, b). In the stroma, MT immunoreactivity was observed on fibroblasts and presented a membranouscytoplasmic pattern of staining (Fig. 3a, b). Low MT immunoreactivivty was seen in patients without lymph node metastases (Fig. 4a, b).

MT immunoreactivity was also determined in the tumor center (older part of the tumor without signs of dynamic growth) and in the tumor front (border, the younger part of the tumor with signs of dynamic growth). The categorization of the central and border parts of the tumor was carried out by means of morphological assessment. The central part of the tumor, with a higher number of apoptotic cells, a small number of fibroblasts and the presence of single macrophages, presented low MT antigen immunoreactivity. The border part of the tumor, was typified by its higher MT antigen immunoreactivity (Fig. 5a, b). Later, Ki67 immunostaining was performed in order to evaluate the rate of proliferation in these areas. The proliferation rate in the areas of the tumors initially defined as central parts was significantly lower when compared with the rate in the areas defined as borders $(P<0.005)$.

In the reference group, MT immunoreactivity was not uniform in the whole tissue of palatine tonsils. In the epithelium, it was exclusively present within the crypts (Fig. 5c), whereas in the lymphoid tissue, it was also observed in the stromal fibroblasts (Fig. 5d).
Vimentin immunoreactivity

Strong immunoreactivity of vimentin antigen was observed within the cancer cells in $31 \%$ of the tissue samples from squamous cell carcinoma and within stromal fibroblasts in $28.9 \%$ of the stromal tissue samples and presented a membranous-cytoplasmic pattern of staining (Figs. 6 and 7). Vimentin immunoreactivity was not observed in the epithelia of the palatine tonsils in the reference samples (not shown).

Comparison of immunoreactivity levels of antigens analyzed in squamous cell carcinoma and stroma samples

The results obtained are presented in Table 3. Statistically significantly higher MT and vimentin immunoreactivity levels were observed in the tissue samples from squamous cell carcinoma than in the stromal tissue samples. No statistically significant differences were observed, however, for CD56, CD57 and vimentin immunoreactivity levels of the tumor when compared with those of the stroma.

Comparison of antigen immunoreactivity levels in squamous cell carcinoma and the reference group

The results are presented in Table 4 . Statistically significantly higher MT and vimentin immunoreactivity levels were
Fig. 4 a Weak MT

immunoreactivity in squamous cell carcinoma derived from a patient without lymph node metastases. Magnification 10×. Bar $50 \mu \mathrm{m}$. b Weak MT immunoreactivity in a patient without lymph node metastases. Magnification 40×. Bar $50 \mu \mathrm{m}$
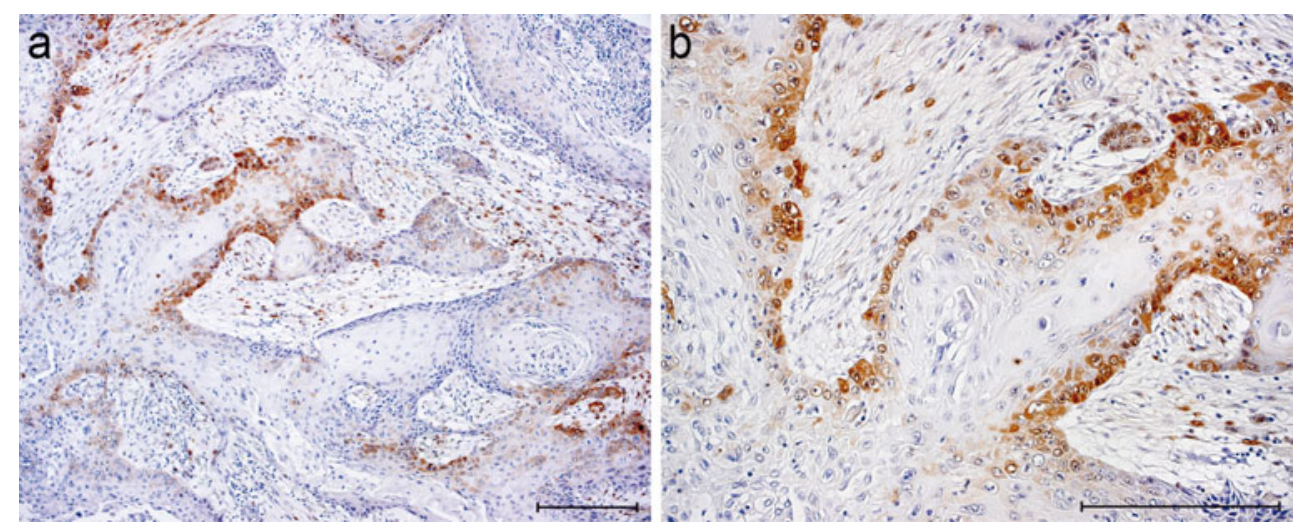
Fig. 5 Analysis of MT immunoreactivity. a High MT antigen immunoreactivity in the border part of the tumor. MTantigen-positive stromal fibroblasts are also present. Magnification $10 \times$. b Low MT antigen immunoreactivity in the central part of the tumor. Magnification $10 \times$. c MT antigen immunoreactivity in the epithelial crypts of a palatine tonsil removed because of chronic tonsillitis.

Magnification $10 \times$. d MTantigen-positive fibroblasts in the stroma of the lymphoid tissue in a palatine tonsil removed because of chronic tonsillitis. Magnification 40x. Bars $50 \mu \mathrm{m}$

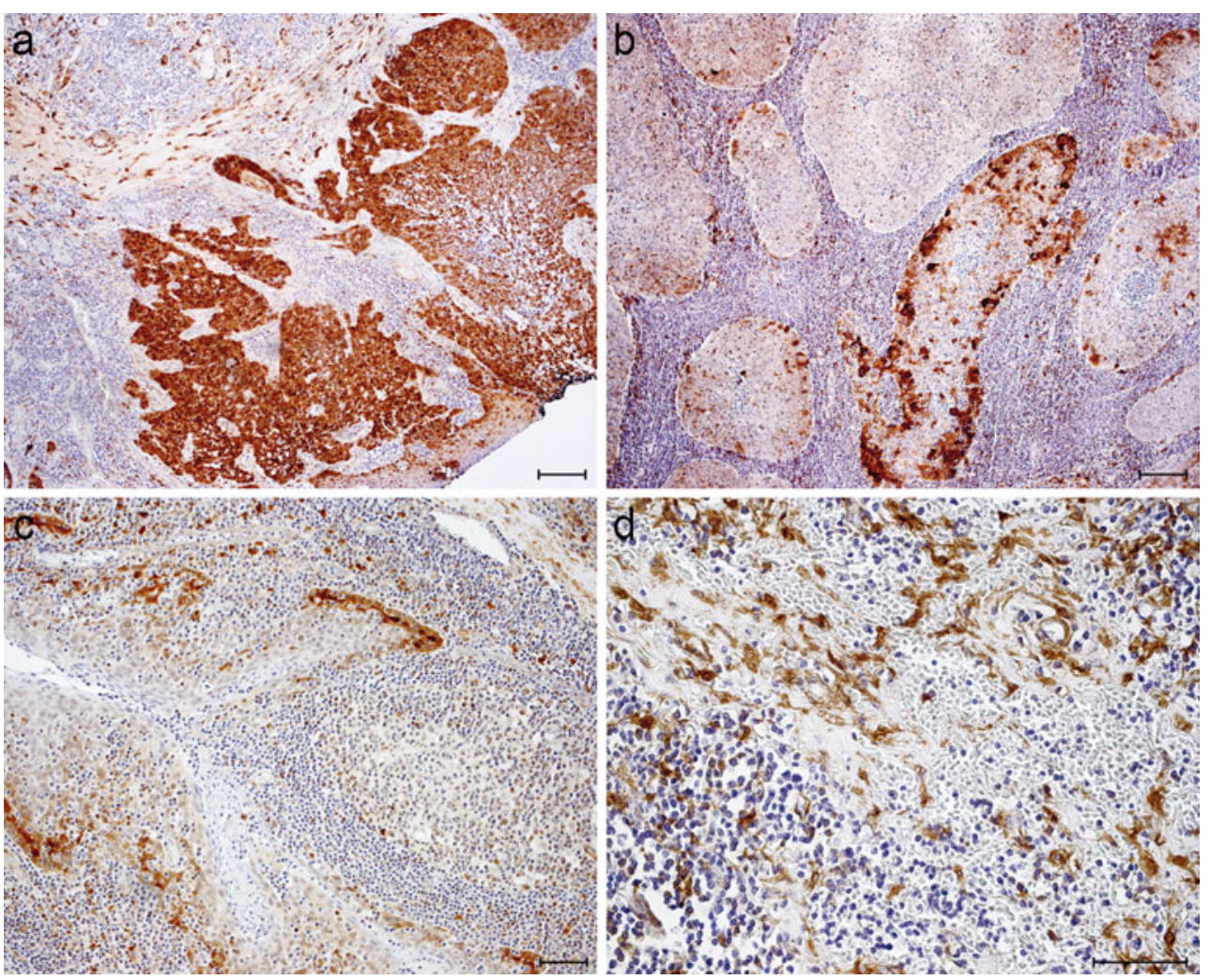

identified in the tissue samples taken from patients with squamous cell carcinoma than in those from the reference group. Statistically significantly higher levels of CD56 and CD57 antigen immunoreactivity were identified in the reference group samples than in the cancer tissue samples.

Comparison of antigen immunoreactivity levels in stroma samples and in reference group samples

The results are presented in Table 5. Statistically significantly higher levels of immunoreactivity of MT, CD56 and CD57 antigens were observed in the reference group samples than in stroma samples. A statistically significantly higher level of vimentin immunoreactivity was observed in the stroma samples than in the reference group samples.

\section{Other comparisons}

The analysis of antigen immunoreactivity levels with respect to tumor grade and stage did not reveal any significant differences.

Statistically significantly higher MT immunoreactivity levels within the tumor cells were identified in patients with the presence of lymph node metastases compared with those patients without such metastases $(P<0.05)$.

Statistically significantly higher vimentin immunoreactivity levels were observed in the tumor stroma in patients
Fig. 6 a, b Intense vimentin immunoreactivity in both the squamous cell carcinoma tissue (arrows) and stromal tissue (stars). Well-demonstrated septa created by the stroma exhibited strong vimentin reactivity. Magnification $10 \times$. Bars $50 \mu \mathrm{m}$
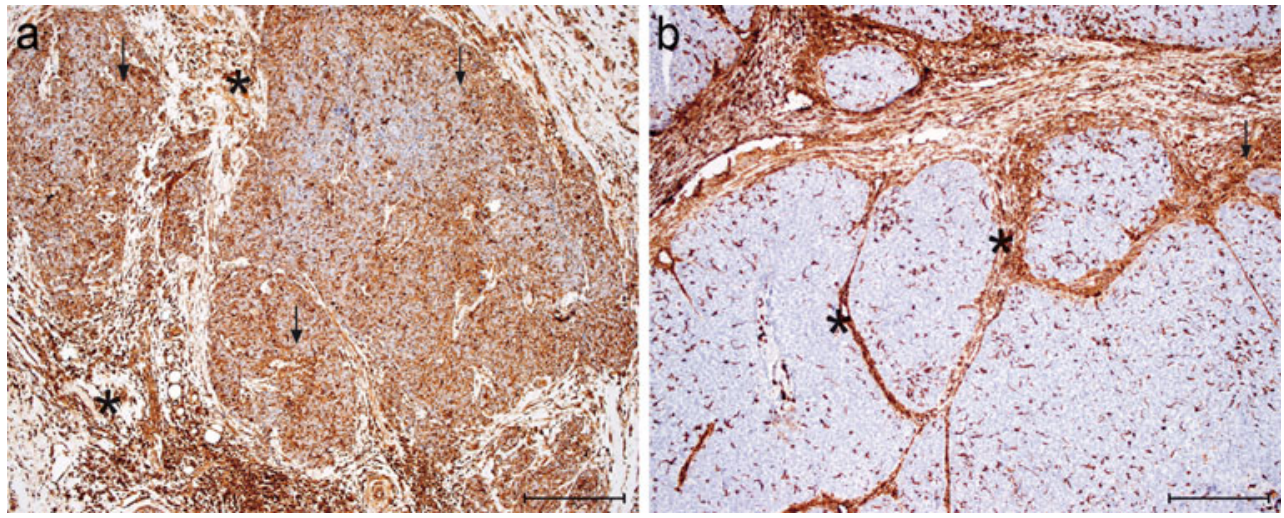


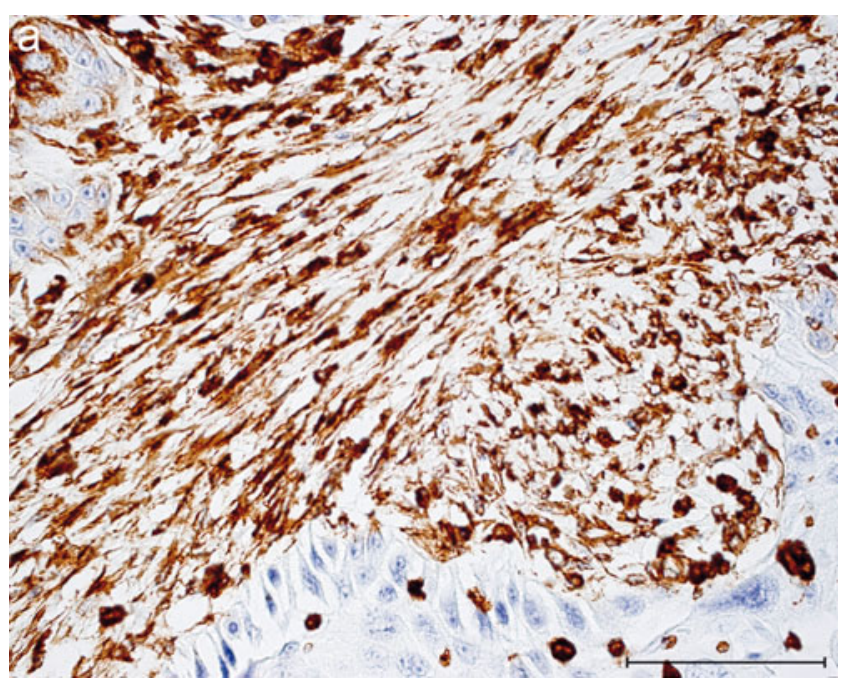

Fig. 7 Immunoreactivity of vimentin antigen with positive fibroblasts in the stroma of squamous cell carcinoma. Magnification 40×. Bar $50 \mu \mathrm{m}$

with advanced tumor size (T3, T4; $P<0.05)$. No statistically significant differences were observed in other analyzed antigens with respect to tumor size.

\section{Discussion}

The MT immunoreactivity levels were statistically significantly higher in the tissue samples from squamous cell carcinoma than in the reference group samples and also higher in the tissue samples from squamous cell carcinoma compared with the stromal samples. Moreover, the stromal fibroblasts exhibited high vimentin and MT immunoreactivity levels. The remodeling of the tumor microenvironment involves all of the tissue adjacent to the tumor and affects both the cells and the extra-cellular matrix and the pattern of expressed proteins. The new pattern of expressed proteins and the transition from the epithelial to mesenchymal phenotype of cells enable the local and general dissemination of the tumor (Dutsch-Wicherek et al. 2005, 2010; Vered et al. 2009; Paccione et al. 2008; Powell et al. 1999). In the

Table 3 Comparison of immunoreactivity levels of antigens analyzed in squamous cell carcinoma and stroma samples (MT metallothionein, NS not significant)

\begin{tabular}{llll}
\hline Antigen & $\begin{array}{l}\text { Squamous cell carcinoma } \\
\text { Median }\left(\mathrm{Q}_{3}-\mathrm{Q}_{1}\right)\end{array}$ & $\begin{array}{l}\text { Stroma } \\
\text { Median }\left(\mathrm{Q}_{3}-\mathrm{Q}_{1}\right)\end{array}$ & $P$-value \\
\hline CD56 & $0(0)$ & $0(0)$ & $\mathrm{NS}$ \\
CD57 & $0(1)$ & $0(0)$ & $\mathrm{NS}$ \\
MT & $2(2)$ & $0(0)$ & $<0.0001$ \\
Vimentin & $1(1)$ & $0(1)$ & $<0.01$ \\
\hline
\end{tabular}

Table 4 Comparison of antigen immunoreactivity levels between tissue samples from squamous cell carcinoma and the reference group, namely chronic tonsillitis epithelia (MT metallothionein)

\begin{tabular}{llll}
\hline Antigen & $\begin{array}{l}\text { Squamous cell carcinoma } \\
\text { Median }\left(\mathrm{Q}_{3}-\mathrm{Q}_{1}\right)\end{array}$ & $\begin{array}{l}\text { Reference group } \\
\text { Median }\left(\mathrm{Q}_{3}-\mathrm{Q}_{1}\right)\end{array}$ & $P$-value \\
\hline CD56 & $0(0)$ & $1.5(2)$ & $<0.001$ \\
CD57 & $0(1)$ & $2.4(3)$ & $<0.001$ \\
MT & $2(2)$ & $0.1(0)$ & $<0.01$ \\
Vimentin & $1(1)$ & $0(0)$ & $<0.001$ \\
\hline
\end{tabular}

present study, the immunoreactivity of vimentin presented an interesting pattern of staining and was strongly expressed within the tumor and its septal architecture. Moreover, the immunoreactivity level of the vimentin antigen in the stroma increased in proportion to the $\mathrm{T}$ stage and was significantly higher in the tumor itself than in the stroma. This differs from results that we found in our previous study concerning adenocarcinoma tissue samples and vimentin expression, where the level of vimentin expression was higher in the stroma than in the tumor and the septal architecture of the tumor was not observed (Dutsch-Wicherek et al. 2010). We did not observe any relationship between the vimentin immunoreactivity in the squamous cell carcinoma cells and the clinicopathological variables; however, in oral squamous cell carcinoma, vimentin was predominantly expressed by cells showing a higher degree of malignancy (Satelli and $\mathrm{Li}$ 2011; de Araujo et al. 1993).

Similar to our previous reports, we observed the heterogeneity of antigens immunoreactivity within the tumor (Jozwicki et al. 2011). MT immunoreactivity was determined in both the tumor center and in the tumor front (the younger part of the tumor with signs of dynamic growth), being significantly higher in the tumor front than in the tumor center. This pattern of expression was similar to that described in other reports with higher expression in the border parts of the tumor and lower expression in the center of the cancer, which is also typified by higher numbers of apoptotic cells, a small number of fibroblasts and the presence of single macrophages (Jayasurya et al. 2000; Sundelin

Table 5 Comparison of CD56, CD57, metallothionein (MT) and vimentin antigens in stroma samples and the reference group, namely epithelia of chronic tonsillitis

\begin{tabular}{llll}
\hline Antigen & $\begin{array}{l}\text { Stroma } \\
\text { Median }\left(\mathrm{Q}_{3}-\mathrm{Q}_{1}\right)\end{array}$ & $\begin{array}{l}\text { Reference group } \\
\text { Median }\left(\mathrm{Q}_{3}-\mathrm{Q}_{1}\right)\end{array}$ & $P$ value \\
\hline CD56 & $0(0)$ & $1.5(2)$ & $<0.001$ \\
CD57 & $0(0)$ & $2.4(3)$ & $<0.001$ \\
MT & $0(0)$ & $0.1(0)$ & $<0.001$ \\
Vimentin & $0(1)$ & $0(0)$ & $<0.001$ \\
\hline
\end{tabular}


et al. 1997; Dutsch-Wicherek et al. 2005). This particular pattern of MT immunoreactivity has been linked to the degree of tumor cell apoptosis (Fas expression). The higher MT expression in the peripheral parts of the tumor has been associated with a reduced number of tumor cells undergoing apoptosis (resulting from MT anti-apoptotic activity) and, at the same time, has been correlated with a higher rate of tumor cell proliferation. Furthermore, the center of the tumor has been found to consist in cancer cells undergoing apoptosis and to show low MT expression (Jayasurya et al. 2000; Sundelin et al. 1997).

As mentioned above, the MT immunoreactivity levels in the present study were significantly higher in the tissue samples from squamous cell carcinoma than in the stroma samples. The presence of MT immunoreactivity in the stroma is intriguing in itself given that this protein has been found in healthy epithelial cells in which it is responsible for epithelial renewal (Dutsch-Wicherek et al. 2005; Popiela et al. 2006; Hellquist 1997). The expression of MT by the stroma raises the question of the real function of this protein in tumor development. In our earlier reports concerning MT expression in cancer and its stroma, we suggested that MT expression might be evoked by the chronic inflammatory process developing in the tumor as a protective mechanism (resistance to apoptosis) of healthy adjacent cells against immune-mediated apoptosis (Dutsch-Wicherek et al. 2005; Popiela et al. 2006). Here, MT immunoreactivity in the stroma was present in the fibroblasts. Cancer-associated fibroblasts play an important role in tumor remodeling and so the MT-positive fibroblasts showing strong vimentin immunoreactivity in the stroma seem to be an important part of the remodeled stroma enabling tumor spread. In the reference group, MT immunoreactivity was observed in the epithelia of the tonsillar crypts of palatine tonsils, whereas in the lymphatic tissue of palatine tonsils, it was observed in the fibroblasts (Fig. 5). Since MT protects the cell against apoptosis and enables proliferation, it is probably a marker of tumor microenvironment remodeling. Additionally, by inhibiting cytotoxic lymphocytes and stimulating the migration of lymphocytes (Sundelin et al. 1997; Yin et al. 2005), MT plays an immunoregulatory role. Borghesi et al. (1996) have speculated that extracellular MT interacts with lymphocyte cellular membranes, modulating their activity, whereas Canpolat and Lynes (2001) have also postulated an important immunomodulating role for MT.

In the present study, we have observed that the MT immunoreactivity level in the cells of squamous cell carcinoma was statistically significantly higher in patients with the presence of lymph node metastases than in patients without such metastases and this agrees with our earlier findings (DutschWicherek et al. 2005). We did not, however, observe this correlation in the adenocarcinoma tissue samples (DutschWicherek et al. 2010). Our findings would seem to confirm that MT expression correlates with poor prognostic factors.
The number of CD56- and CD57-antigen-positive lymphocytes identified in the tissue samples both from squamous cell carcinoma and from the stroma was statistically significantly lower than that in the reference group in the present study. No differences were however seen in the number of CD56+ and CD57+ lymphocytes between the tumor and the stroma. The level of anti-cancer response in our study, as shown by the CD56- and CD57-expressing lymphocytes, seemed to remain at a comparable level in both the tumor and its stroma. All of these findings indicate a lack of anti-cancer immune response in squamous cell carcinoma and in the stroma and confirm the suppressive profile of the tumor microenvironment as represented by the deficit of CD56 and CD57 effector cells.

In conclusion, MT expression by tumor cells is associated with an aggressive phenotype of the tumor and its ability to create metastases.

Open Access This article is distributed under the terms of the Creative Commons Attribution License, which permits any use, distribution and reproduction in any medium, provided the original author(s) and the source are credited.

\section{References}

Acevedo VD, Gangula RD, Freeman KW, Li R, Zhang Y, Wang F, Ayala GE, Peterson LE, Ittmann M, Spencer DM (2007) Inducible FGFR1 activation leads to irreversible prostate adenocarcinoma and an epithelial-to-mesenchymal transition. Cancer Cell 12:559-571

Apostolova MD, Cherian MG (2000) Nuclear localization of metallothionein during cell proliferation and differentiation. Cell Mol Biol 46:347-356

Araujo VC de, Pinto Júnior DS, Sousa SO de, Nunes FD, Araujo NS de (1993) Vimentin in oral squamous cell carcinoma. Eur Arch Otorhinolaryngol 250:105-109

Borghesi LA, Youn J, Olson EA, Lynes MA (1996) Interactions of metallothionein with murine lymphocytes: plasma membrane binding and proliferation. Toxicology 108:129-140

Canpolat E, Lynes MA (2001) In vivo manipulation of endogenous metallothionein with a monoclonal antibody enhances a Tdependent humoral immune response. Toxicol Sci 62:61-70

Cherian MG, Jayasurya A, Bay BH (2003) Metallothioneins in human tumors and potential roles in carcinogenesis. Mutat Res 33:201-209

De Wever O, Mareel M (2005) Role of tissue stroma in cancer cell invasion. J Pathol 200:429-447

Dutsch-Wicherek M, Popiela TJ, Klimek M, Rudnicka-Sosin L, Wicherek L, Oudinet JP, Skladzien J, Tomaszewska R (2005) Metallothioneinstroma reaction in tumor adjacent healthy tissue in head and neck squamous cell carcinoma and breast adenocarcinoma. Neuro Endocrinol Lett 26:567-574

Dutsch-Wicherek M, Sikora J, Tomaszewska R (2008) The possible biological role of metallothionein in apoptosis. Front Biosci 13:4029-4038

Dutsch-Wicherek M, Lazar A, Tomaszewska R (2010) The potential role of MT and vimentin immunoreactivity in the remodeling of the microenvironment of parotid adenocarcinoma. Cancer Microenviron 4:105-113

Dvorak HF (1986) Tumors: wounds that do not heal. Similarities between tumor stroma generation and wound healing. N Engl $\mathrm{J}$ Med 315:1650-1659 
Grunert S, Jechlinger M, Beug H (2003) Diverse cellular and molecular mechanisms contribute to epithelial plasticity and metastasis. Nat Rev Mol Cell Biol 4:657-665

Hayward SW, Wang Y, Cao M, Hom YK, Zhang B, Grossfeld GD, Sudilovsky D, Cunha GR (2001) Malignant transformation in a nontumorigenic human prostatic epithelial cell line. Cancer Res 61:8135-8142

Hellquist HB (1997) Apoptosis in epithelial hyperplastic laryngeal lesions. Acta Otolaryngol Suppl 527:25-29

Hugo H, Ackland ML, Blick T, Lawrence MG, Clements JA, Williams ED, Thompson EW (2007) Epithelial-mesenchymal and mesenchymal-epithelial transitions in carcinoma progression. J Cell Physiol 213:374-383

Jayasurya A, Bay BH, Yap WM, Tan NG (2000) Correlation of metallothionein expression with apoptosis in nasopharyngeal carcinoma. Br J Cancer 82:1198-1203

Jozwicki W, Windorbska W, Brozyna AA, Jochymski C, Basta P, Sikora J, Stasienko E, Dutsch-Wicherek M, Koper K, Wicherek L (2011) The analysis of receptor-binding cancer antigen expressed on SiSo cells (RCAS1) immunoreactivity within the microenvironment of the ovarian cancer lesion relative to the applied therapeutic strategy. Cell Tissue Res 345:405-414

Klaassen CD, Liu J, Choudhuri S (1999) Metallothionein: an intracellular protein to protect against cadmium toxicity. Annu Rev Pharmacol Toxicol 39:267-294

Kuss I, Hathaway B, Ferris RL, Gooding W, Whiteside TL (2004) Decreased absolute counts of $\mathrm{T}$ lymphocyte subsets and their relation to disease in squamous cell carcinoma of the head and neck. Clin Cancer Res 10:3755-3762

Lorusso G, Ruegg C (2008) The tumor microenvironment and its contribution to tumor evolution toward metastasis. Histochem Cell Biol 130:1091-1103

Okada T, Iiai T, Kawachi Y, Moroda T, Takii Y, Hatakeyama K, Abo T (1995) Origin of CD57+ T cells which increase at tumor sites in patients with colorectal cancer. Clin Exp Immunol 102:159-166

Orimo A, Gupta PB, Sgroi DC, Arenzana-Seisdedos F, Delaunay T, Naeem R, Carey VJ, Richardson AL, Weinberg RA (2005) Stromal fibroblasts present in invasive human breast carcinomas promote tumor growth and angiogenesis through elevated SDF-1/CXCL12 secretion. Cell 121:335-348

Paccione RJ, Miyazaki H, Patel V, Waseem A, Gutkind JS, Zehner ZE, Yeudall WA (2008) Keratin down-regulation in vimentin-positive cancer cells is reversible by vimentin RNA interference, which inhibits growth and motility. Mol Cancer Ther 7:2894-2903
Popiela TJ, Rudnicka-Sosin L, Dutsch-Wicherek M, Klimek M, Basta P, Galazka K, Wicherek L (2006) The metallothionein and RCAS1 expression analysis in breast cancer and adjacent tissue regarding the immune cells presence and their activity. Neuro Endocrinol Lett 27:786-794

Powell DW, Mifflin RC, Valentich JD, Crowe SE, Saada JI, West AB (1999) Myofibroblasts. I. Paracrine cells important in health and disease. Am J Physiol 277:C1-C9

Sallusto F, Geminat J, Lanzavecchia A (2004) Central memory and effector memory $\mathrm{T}$ cell subsets: function, generation, and maintenance. Annu Rev Immunol 22:745-763

Satelli A, Li S (2011) Vimentin in cancer and its potential as a molecular target for cancer therapy. Cell Mol Life Sci 68:30333046

Satoh M, Seki S, Hashimoto W, Ogasawara K, Kobayashi T, Kumagai K, Matsuno S, Takeda K (1996) Cytotoxic gammadelta or alphabeta $\mathrm{T}$ cells with a natural killer cell marker, CD56, induced from human peripheral blood lymphocytes by a combination of IL-12 and IL-2. J Immunol 157:3886-3892

Shimoda R, Achanzar WE, Qu W, Nagamine T, Takagi H, Mori M, Waalkes MP (2003) Metallothionein is a potential negative regulator of apoptosis. Toxicol Sci 73:294-300

Sundelin K, Jadner M, Norberg-Spaak L, Davidsson A, Hellquist HB (1997) Metallothionein and Fas (CD95) are expressed in squamous cell carcinoma of the tongue. Eur J Cancer 33:1860 1864

Takii Y, Hashimoto S, Iiai T, Watanabe H, Hatakeyama K, Abo T (1994) Increase of the proportion of granulated CD56+ T cells in patients with malignancy. J Clin Exp Immunol 97:522-527

Theocharis SE, Margeli AP, Klijanienko JT, Kouraklis GP (2004) 617 Metallothionein expression in human neoplasia. Histopathology 618:103-118

Vered M, Allon I, Buchner A, Dayan D (2009) Stromal myofibroblasts accompany modifications in the epithelial phenotype of tongue dysplastic and malignant lesions. Cancer Microenviron 2:49-57

Witz IP (2009) The tumor microenvironment: the making of a paradigm. Cancer Microenviron 2:S9-S17

Xu J, Wang R, Xie ZH, Odero-Marah V, Pathak S, Multani A, Chung LW, Zhau HE (2006) Prostate cancer metastasis: role of the host microenvironment in promoting epithelial to mesenchymal transition and increased bone and adrenal gland metastasis. Prostate 66:1664-1673

Yin X, Knecht DA, Lynes MA (2005) Metallothionein mediates leukocyte chemotaxis. BMC Immunol 6:21 